\title{
Using Smartphones to Profile Mobility Patterns in a Living Lab for the Transition to E-mobility
}

\author{
Francesca Cellina ${ }^{1}$, Anna Förster ${ }^{1}$, Davide Rivola ${ }^{1}$, Luca Pampuri ${ }^{1}$, \\ Roman Rudel ${ }^{1}$, and Andrea Emilio Rizzoli ${ }^{2}$ \\ 1 SUPSI - University of Applied Sciences of Southern Switzerland \\ \{francesca.cellina, anna.foerster, davide.rivola, luca.pampuri, \\ roman.rudel\}@supsi.ch \\ 2 IDSIA, USI-SUPSI, Switzerland \\ andrea@idsia.ch
}

\begin{abstract}
The diffusion of electric vehicles is currently the most promising opportunity to reduce the dependency on fossil fuel in the mobility sector. However, the adoption of the electric vehicles seems to be still hindered by psychological and behavioural barriers. Thus, in order to understand and to foster the transition towards more sustainable mobility styles, it becomes essential to adopt an inter-disciplinary approach. In this framework, the e-mobiliTI project was launched in late 2012 in Southern Switzerland. It aims at understanding the potential for transition in the mobility system at the local level, with a special focus on electric mobility. The project builds upon a small living lab made up of around twenty families, who will be monitored in all their trips through smart mobile devices, in order to get quantitative data, and through focus groups, in order to get qualitative data and perceptions.

Here we discuss the major challenge in the initial stage of the emobiliTI project, that is the gathering of reliable and high-quality data on users' behaviour. We describe the automatic tracking system, and the data processing and the qualitative assessment approach and comment on the overall performances of the living lab experiment.
\end{abstract}

Keywords: personal mobility tracking, smartphones, mobility living lab, mobility transition.

\section{Introduction and Motivation}

The diffusion of electric vehicles is currently the most promising opportunity to reduce the dependency on fossil fuels in the mobility sector and to pave the way for the transition to a more sustainable society.

In the last few years technology made remarkable progresses. In Switzerland, nowadays more than $80 \%$ of the demand of personal mobility activities and patterns could be covered by electric vehicles: the 2010 Swiss Transport and mobility census in fact indicates that $84 \%$ of the trips are shorter than 10 kilometers and that $73 \%$ of them are shorter than 5 kilometres [1. Currently there are no monetary incentives at the national level for electric vehicles, since the Swiss

J. Hřebíček et al. (Eds.): ISESS 2013, IFIP AICT 413, pp. 154-163, 2013.

(C) IFIP International Federation for Information Processing 2013 
government believes their introduction should only be driven by the market [2. However, in Canton Ticino, located in Southern Switzerland, the government is on the verge to approve an incentive scheme for electric and hybrid vehicles, allowing a reduction of the vehicle sale price up to 10'000 CHF.In addition, the network for public charging stations for electric vehicles is already densely distributed all over the urbanised areas. The charging infrastructure will be furthermore supported by a private initiative, named "EVite", which is planning to install up to 250 public fast-charge stations in Switzerland. Finally, the cost of electric vehicles has worldwide dramatically decreased, up to the point that in a few years it is expected to become equivalent to conventional fossil fuelled vehicles (see for example [3]).

Therefore, all the conditions seem to favour a transition to electric mobility, but this is going to be a large scale and long-term process. Transitions of this type are determined by a wide set of factors, acting together. In the case of electric vehicles, their adoption seems to be still hindered by psychological and behavioural barriers (e.g. issues such as the breadth of the autonomy range, the availability and diffusion of recharge points, the performance, see [4, [5], 6], [7]). Thus, in order to understand and, at a later stage, to foster the transition towards more sustainable mobility styles, it becomes essential to adopt an inter-disciplinary approach, able to integrate psychology, sociology, cultural and spatial planning aspects, alongside the technology and engineering aspects.

Among various factors, consumer behaviour is one of the most influential ones, at least as important as technology $([8])$. Adopting a socio-technical perspective $([9],[10])$ and an actor-based approach allows a deeper and more effective understanding of this fundamental phenomenon. The living lab research approach ([1]) offers promising perspectives, since it allows real life users to explore and experience new technologies in complex, real-world settings.

In the remainder of this paper, first we present the e-mobiliTI project context (Section 2), then we describe the smart-phone app we developed to track user behaviour and collect user feedback (Section 3). We then quickly describe the approach for profiling user behaviour and understanding the drivers for change (Sections 4), and finally we critically discuss the major challenges we will face during the project (Section 5).

\section{The e-mobilitI Living Lab Experiment}

The e-mobiliTI project is funded and coordinated by the University of Applied Sciences of Southern Switzerland (SUPSI) and it is supported by regional authorities, with the final aim of elaborating policy guidelines, incentives, procedures and strategies for a transition in the mobility sector at the urban level.

\subsection{Background and Approach}

The e-mobiliTI project adopts an inter-disciplinary, bottom-up approach, aimed at exploring the potential effects and benefits of innovative mobility technologies and options through experimentation and direct interaction with the end-users. 
Similar socio-technical experiments were held in the past, in the framework of the Strategic Niche Management (SNM) approach, which was developed in the late Nineties at the European level and was successfully applied in the transportation sector ([12, [13]). More recently, similar approaches started being indicated as Living lab processes, intended as inter-disciplinary and user-centric methodologies, that, involving real users in actual living environments, allow to develop, deploy and test new technologies and strategies capable of responding to the complex and evolving needs of the present complex society 1 .

The SNM and Living lab methodologies are especially effective in systems offering environmental and social benefits which are undervalued on the market 13. in particular by single individuals, as in the transportation sector. Successful experiences in this sector were for example gathered in Denmark since 2009, with the TryAnEV/Insero E-mobility project 2. For less recent experiences, one can for example refer to [14].

\subsection{Research Objectives and Expected Outcome}

Project activities are being developed in two main phases (see Figure 11): in Spring 2013 the families involved in the living lab are monitored in order to identify their present mobility patterns and styles, while in Spring 2014 they are monitored while testing and experiencing new mobility options, namely electric bicycles and cars as well as car-sharing (Mobility), bike-sharing (Velopass) and season tickets for public means of transportation (Arcobaleno).

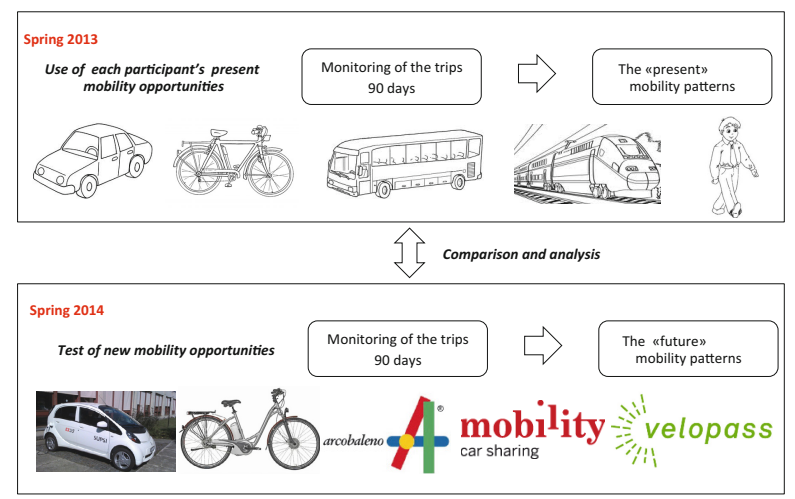

Fig. 1. The two phases of the e-mobiliTI experiment

Providing the participants with the new mobility options, e-mobiliTI removes the present main barrier to the diffusion of electric cars and bicycles, that is their

${ }^{1}$ MIT living lab website http://livinglabs.mit.edu/ retrieved on April 24th, 2013.

${ }^{2}$ Nordic Sustainable Logistics Network website http://www.noslone.com/News/Details.aspx?id=70, retrieved on May 13rd, 2013. 
price, and creates a niche in which we can analyse the participants behaviour with respect to the other barriers, such as: range, recharge and performance for the electric cars; physical effort and safety for e-bikes; status-symbol and rigidity for vehicle sharing; rigidity and punctuality for public transport.

Comparing the mobility patterns and styles observed during the two monitoring phases allows us to test the following hypotheses:

1. barriers affecting the use of electric vehicles are mainly psychological and can be removed through experience - or, in other words: using an electric vehicle or bicycle does not require major sacrifices or reductions in the present single individuals well-being;

2. having an electric vehicle or bicycle at one's disposal acts as a leverage for a general transformation of the individual mobility patterns (slow mobility, public transport and use of shared mobility services), instead of merely acting as a pure substitute of conventional vehicles and bicycles.

In order to identify the mobility patterns and styles of the participants in the living lab, during the two phases we rely on both quantitative automatic data-gathering techniques and on group discussions and single interviews for the analysis and review of qualitative data. Automatic data-gathering is performed by means of a specifically developed smart-phone application (app) that relies on GPS tracks and on Bluetooth sensors placed on vehicles and bikes to identify transport modes [15. The app posts GPS and Bluetooth data to a centrally hosted database, where data are post-processed in order to make them available to further processing by using data classification techniques aiming at the identification of mobility patterns and styles. Such users profiling also considers information gathered through qualitative discussion groups, mainly focus-groups: the latter suggests hypotheses on styles and patterns to be verified by means of the data mining classification. From the computer science point of view, thus, the e-mobiliTI project has the further objective of effectively integrating the quantitative information produced by the smart mobile devices to the qualitative information produced by the focus groups.

\subsection{Selection of the Participants}

For the setting of the experiment, we selected the Lugano region in the Southern Switzerland Ticino Canton, i.e. an urban conurbation of around 135'000 inhabitants. It is particularly interesting because, even though it is often congested and characterised by frequent traffic jams, it already offers alternatives to traditional private owned fossil fuelled vehicles, such as an efficient public transportation network and car-sharing and bike-sharing services.

Selecting the participating families is a key and delicate activity. We performed it by means of a public open call, promoted via the local mass-media (newspapers, television, radio) and via flyers distributed all over the city: therefore, participants come from a process of self-nomination of the interested people.

The public call was open to generic families, i.e. either single individuals or couples or couples with (older) children. More than 350 candidates answered the 
call: a much higher number than we initially expected, reflecting a very high interest also in the common people for new mobility technologies and options. The selection process aimed at identifying an homogeneous group of subjects based on the place of living, on the demographic features of the family (single, couple, couple with (older) children) and on the present use of mobility options. This process allowed us to identify a set of sixteen families, for a total number of thirty persons actively involved.

\section{$3 \quad$ Smart Mobile Devices}

In order to capture as precisely as possible and as many as possible trips from our users, we turned our attention to smartphone based gathering. This approach turned out to be a bless and a curse at the same time: a bless, because we are able to gather really a vast amount of data from different users for long periods of time; a curse, because the usage of the smartphone based application proved to be quite complex for many users. In this section, we detail the general architecture of our solution and present the main functionalities of the application.

\subsection{General Architecture}

The general architecture of our system, which we also call the e-mobiliTI app, is presented in Figure 2. It consists of the smartphone based application, which communicates to the central server to send the gathered data and to receive the daily reports. The application gathers GPS and Bluetooth data every 1-3 minutes, depending on how fast the user is moving (1 min when moving fast). If the smartphone is not moving at all, the GPS and Bluetooth sampling is interrupted to save the battery. This data is sent regularly to the central server, which stores the data. Once a day, at a user-defined time, the server evaluates all available data, automatically identifies moving segments of the user together with the used vehicle and sends these segments in the form of a daily report back to the smartphone. There, the user is notified and can review all segments and correct them, where necessary.

Additionally to the smartphone (currently running Android OS), each user's car is equipped with a Bluetooth tag. Any Bluetooth device can be used, but the system requires that the tag is always on. A good option is the stick'n'find Bluetooth tag ${ }^{3}$ or the Blue Solar car kit from MrHandsFree 4 , which are always on and are very energy efficient.

\subsection{Automatic Recognition of Trip Segments}

One of the most important functionalities of the system is its ability to recognise the movements of the users automatically and to break them down into segments. According to the commonly used terminology, we define:

\footnotetext{
3 http://www.sticknfind.com

4 http://www.mrhandsfree.com
} 


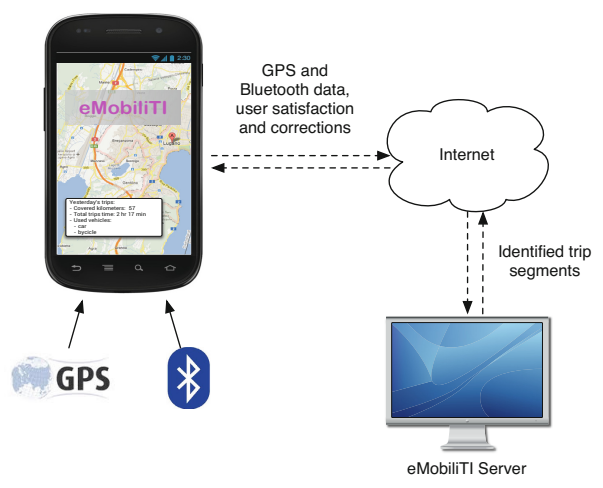

Fig. 2. The general architecture of our smartphone based solution. The e-mobiliTI app gathers GPS and Bluetooth data and sends them to the central server. The server analyses the data daily and sends to the user all identified trip segments. The user can thus make corrections to the properties of the trip and give her overall satisfaction level, which are again sent to the server for backup.

- Trip: a movement of the user starting at her home and ending also there. There are also other possible definitions of a trip, however this one is the most well defined and non-ambiguous one.

- Segment: part of a trip, which can be associated with a single vehicle used (a bus, a car, on foot, etc.), separated by stop points. A trip can consist of a single segment, but usually it consists of more than one segment.

- Stop: it is a humanly defined stop point on a trip, corresponding to some short or long interrupt to the trip. For example, stopping at the gas station, to chat with a friend, at the bakery etc. However, it also includes long stops, such as for whole day work in the office.

Thus, a trip is a sequence of alternating stops and segments, where both the start and the end places are at home. Our system is able to recognise all there levels of the trip: the stops, the segments and the trips themselves. The algorithm is based on the following steps:

- Recognize stop points by identifying GPS points very close to each other (at most 100 meters apart) for at least 2 minutes.

- Segment the data into stops (as identified above) and segments.

- Validate the segments with some minimum requirements for distance and velocity. Delete all failed segments by merging them together.

- Validate the stops between the segments again by deleting them first and analysing the newly formed segments. If the segment is a valid one (see above), the stop remains deleted. If the segment does not fulfil the requirements any more, put back the stop.

The vehicle is recognised by thresholds on the mean and maximum speeds in a segment. The Bluetooth data is used to recognise the own cars of the users and to differentiate them from public transport or other cars. 
The developed algorithm is quite flexible and is able to identify short stops (such as changing the bus at a bus stop), while at the same removing artefacts such as stopping at the traffic light. Of course, not all artefacts are removed and there remain a certain number of segments, which are shorter than expected.

\subsection{Graphical User Interface and Functionalities}

The app graphical user interface for the review of the trips by the users is presented in Figure 3. The left-most picture (a) shows the daily survey with the general daily satisfaction level and note and all identified segments for that day. (b) presents the details of one segment, with start and end times, total distance, a map with start and end places and the recognised vehicle. Also, the start and end places can be associated with labels (e.g. home, office, school, bus stop, etc.) and the user can also enter her motivation for the trip, the number of people she is travelling with and the satisfaction for the segment only. Once the user completes the report, the updated data is sent back to the server for final storage. Of course, the user can change also old surveys and segments or complete the survey later than the predefined time.

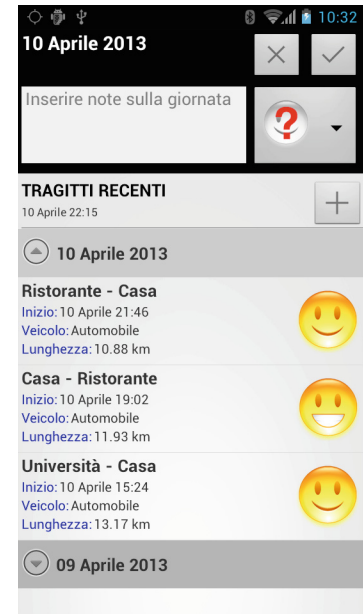

(a)

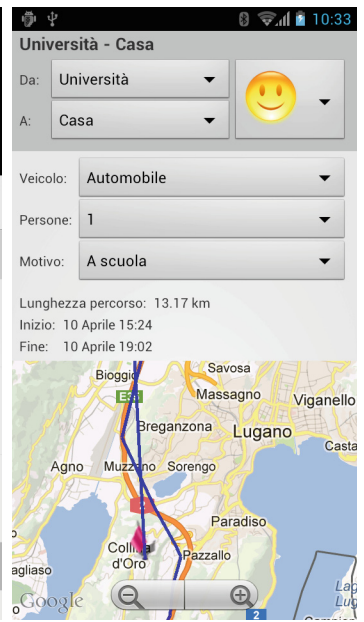

(b)

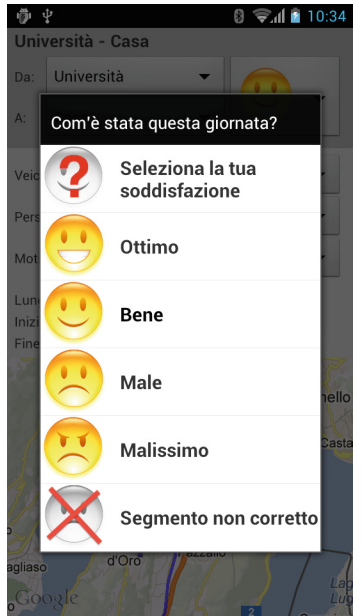

(c)

Fig. 3. The graphical user interface to review the movements of the day and to insert details and satisfaction level

\subsection{Practical Notes}

The implementation on the smartphone is straightforward, but requires a lot of additional functionalities to make it user-friendly at one side and robust on the other side. The main challenge comes from the very different technological skills of the users and their attitude towards the experiment. 
In the implementation we also considered the following requirements: when powering on the phone the app had to be automatically started; GPS and Bluetooth had to be also turned on at start-up and kept active; the notification of the alarm signal for the daily report had to remain loud even when the smartphone was silenced; failed data transmission to the servers because of lack of signal had to be queued up for later re-send; all entered data needed to be saved without additional user confirmation; the phone settings were corrected not to allow data roaming, but to always allow $3 \mathrm{G}$ data connection (for cost efficiency), and not to use any energy saving options (as they are faulty on many Android phones).

\section{User Profiling and Evaluation}

The collected data on the various mobility choices of all participants in the experiment will then be processed in order to discover and understand how they make their daily personal mobility choices. Given the limited size of the sample, this part of our study is aimed at understanding how a specific person chooses a specific mobility option as a function of various possible influencing factors, such as distance, tightness of schedule, reason for the trip, number of intermediate stops, the weather conditions and so on. For each user, her daily trips will be analysed, looking for patterns, such as the choice of a given mean of transport, or a combination of means, to a specific purpose. We will therefore build a mobility profile for each user, and we will then also look for similarities among all users, on the basis of their typology.

During the second phase of the project, the same users will be monitored, but this time their travel options will be wider, as they will have the choice of opting for public transport, shared mobility and electric mobility options. The data collected during this part of the experiment will also be used to create the users' profiles. The new profiles will be then compared with the old ones, i.e. those obtained before the introduction of new mobility options, and the objective of the comparison will be the identification of the most relevant factors influencing a transport choice change, provided a given degree of profile similarity among the users.

\section{Discussion and Conclusions}

The e-mobiliTI project relies on the strong involvement of the users, the key components of the living lab, in order to evaluate how a transition to sustainable mobility styles could happen. Such a project is therefore fraught with problems which are common to this type of projects, namely: users' motivation to actively participate in the project can dwindle; automated data collection covers only quantitative data, while qualitative data collection presents some challenges; finally, the size of the sample is small, and thus the general validity of the output of the project, the policy guidelines, can be criticised.

The users's attitude during the project is probably the most important challenge, since it cannot be tackled from a purely technical point of view. Literature on the living lab approach in fact implicitly assumes that the users are 
cheap or unpaid contributors, motivated by the hope that their participation will solve their problems or lead to better policy-making [16. Will our living labs achieve and sustain the necessary level of user mobilisation and cooperation in the absence of stronger incentives [17]? Will we be able enough in motivating the participants as active co-creators of the transition towards a new mobility system? This challenge is particularly relevant during the first e-mobiliTI monitoring phase, when they are asked to use their own mobility options, thus being little interested in getting reports of their trips. We expect that probably, when in the second monitoring phase they will be allowed to try new mobility options, their curiosity respect to the daily survey will increase.

Provided that users keep an high involvement in the project and keep the emobiliTI app active at all times and regularly collect data, certain types of information, such as the reason for the trip, the number of people travelling together and the overall satisfaction for the trip, can only come from personal information enrichments, voluntarily entered by the participants to the e-mobiliTI living lab. For this reason, the $a p p$ provides the users with a daily report of their segments, which, besides offering them the possibility to correct erroneous trips, allows them to insert the cited qualitative information. However, this is not enough to grasp the full reasons for their behaviour. That is why we believe that physical, personal contact during focus groups and single interviews is essential to fully understand their mobility patterns and styles.

Finally, different users may face different "real-world" situations, or even interpret them in different ways, according to their personal perception and value systems [18. Thus, in order to get enough different perspectives, it is important to involve a large enough number of users, which, in some cases, might be burdensome and costly. In our experiment, due to limits in the available budget, the number of participating families is very small: therefore, no general representativeness can be attributed to the mobility patterns and lifestyle observed within the e-mobiliTI living lab. The project in fact cannot provide results capable of representing the whole Lugano region inhabitants behaviour and perceptions. While we acknowledge this limitation, on the other hand the information gathered during the project lifetime can provide a starting point for the niche characterisation and suggest the initial actions to be taken to favour the transition towards different mobility lifestyles.

\section{References}

1. Office fédéral de la statistique (OFS), Office fédéral du développement territorial (ARE): La mobilité Suisse - Rèsultats du Microrecensement Mobilité et transports 2010. Technical report, Neuchàtel et Berne (2012)

2. IA-HEV: Hybrid and electric vehicles annual report. Technical report, IEA (2012)

3. World Bank (PRTM Management Consultants): The China New Energy Vehicles Program: challenges and opportunities. Technical report, Bejing (2011)

4. Everett, A., Burgess, M., Harris, M., Mansbridge, S., Lewis, E., Walsh, C., Carrol, S.: Initial Findings from the Ultra Low Carbon Vehicle Demonstrator Programme: How quickly did users adapt? - A review of preliminary usage and perception data from the first three months of vehicle trials. Technical report (2011) 
5. Turrentine, T., Garas, A., Lentz, J., Woodjack, J.: The UC Davis MINI E Consumer Study. Technical Report Research Report UCD-ITS-RR-11-05, 2011, Institute of Transportation Studies, University of California, Davis (2011)

6. Deloitte: Gaining traction - A customer view of electric vehicle mass adoption in the U.S. automotive market. Technical report (2010)

7. Cocron, P., Bühler, F., Neumann, I., Franke, T., Krems, J., Schwalm, M., Keinath, A.: Methods of evaluating electric vehicles from a user's perspective - the MINI E field trial in berlin. IET Intelligent Transport Systems 5(2), 127-133 (2011)

8. Geels, F., Kemp, R., Dudley, G., Lyons, G.: Automobility in transition? A sociotechnical analysis of sustainable transport. Routledge (2011)

9. Schot, J.: Towards new forms of participatory technology development. Technology Analysis \& Strategic Management 13(1), 39-52 (2001)

10. Geels, F., Schot, J.: Typology of sociotechnical transition pathways. Research Policy 36(3), 399-417 (2007)

11. Higgins, A., Klein, S.: Introduction to the living lab approach. In: Tan, Y.H., et al. (eds.) Accelerating Global Supply Chains with IT-Innovation, Springer, Heidelberg (2011)

12. Kemp, R., Schot, J., Hoogma, R.: Regime shifts through processes of niche formation. the approach of strategic niche management. Technology Analysis \& Strategic Management 10(2), 175-195 (1998)

13. Kemp, R., Truffer, B., Harms, S.: Strategic niche management for sustainable mobility. In: Rennings, K., Hohmeyer, O., Ottinger, R. (eds.) Social costs and sustainable mobility: strategies and experiences in Europe and the United States, pp. 167-188. Physica-Verlag, Heidelberg (2000)

14. Hoogma, R., Kemp, R., Schot, J., Truffer, B.: Experimenting for sustainable transport: the approach of strategic niche management. Spon Press, London (2002)

15. Förster, A., Cabrini, M., Garg, K., Giordano, S.: Understanding and optimizing human mobility with smart phones. In: Poster at the International Conference on ICT for Sustainability, Zurich, Switzerland (2013)

16. Mulder, I., Stappers, P.J.: Co-creating in practice: results and challenges. In: Collaborative Innovation: Emerging Technologies, Environments and Communities (Proceedings of the 15th International Conference on Concurrent Enterprising, ICE 2009, Leiden, The Netherlands, June 22-24, Centre for Concurrent Enterprise, Nottingham (2009)

17. Dutilleul, B., Birrer, F.A., Mensink, W.: Unpacking european living labs: analysing innovation's social dimensions. Central European Journal of Public Policy 4(1), 6085 (2010)

18. Bergvall-Kåreborn, B., Ihlström Eriksson, C., Ståhlbröst, A., Svensson, J.: A milieu for innovation-defining living labs. In: 2nd ISPIM Innovation Symposium, New York (2009) 